\title{
Maternal smoking and the risk of still birth: systematic review and meta-analysis
}

\author{
Takawira C Marufu ${ }^{1}$, Anand Ahankari ${ }^{1,2}$, Tim Coleman $^{3}$ and Sarah Lewis ${ }^{1 *}$
}

\begin{abstract}
Background: Smoking in pregnancy is known to be associated with a range of adverse pregnancy outcomes, yet there is a high prevalence of smoking among pregnant women in many countries, and it remains a major public health concern. We have conducted a systematic review and meta-analysis to provide contemporary estimates of the association between maternal smoking in pregnancy and the risk of stillbirth.

Methods: We searched four databases namely MEDLINE, EMBASE, Psych Info and Web of Science for all relevant original studies published until $31^{\text {st }}$ December 2012. We included observational studies that measured the association between maternal smoking during pregnancy and the risk of stillbirth.

Results: 1766 studies were screened for title analysis, of which 34 papers $(21$ cohorts, 8 case controls and 5 cross sectional studies) met the inclusion criteria. In meta-analysis smoking during pregnancy was significantly associated with a $47 \%$ increase in the odds of stillbirth (OR 1.47, 95\% Cl 1.37, 1.57, p < 0.0001). In subgroup analysis, smoking $1-9 \mathrm{cig} /$ day and $\geq 10 \mathrm{cig} /$ day was associated with an $9 \%$ and $52 \%$ increase in the odds of stillbirth respectively. Subsequently, studies defining stillbirth at $\geq 20$ weeks demonstrated a $43 \%$ increase in odds for smoking mothers compared to mothers who do not smoke, $(\mathrm{OR} 1.43,95 \% \mathrm{Cl} 1.32,1.54, \mathrm{p}<0.0001)$, whereas studies with stillbirth defined at $\geq 24$ weeks and $\geq 28$ weeks showed $58 \%$ and $33 \%$ increase in the odds of stillbirth respectively.

Conclusion: Our review confirms a dose-response effect of maternal smoking in pregnancy on risk of stillbirth. To minimise the risk of stillbirth, reducing current smoking prevalence in pregnancy should continue to be a key public health high priority.
\end{abstract}

\section{Background}

Smoking in pregnancy is a major public health problem in the developed countries [1]. The World Health Organisation (WHO) predicts that this will reach 'epidemic' proportions in developing countries in the near future [1]. Within the current challenging economic climate in many countries, smoking in pregnancy imposes a significant burden on population health and resources, and is associated with a range of poor outcomes for both mother and child, such as ectopic pregnancy, miscarriage, placental abruption, preterm birth and low birth weight $[2,3]$. The harmful effects of tobacco smoke exposure in pregnancy can be avoided [4] and it is one of

\footnotetext{
*Correspondence: Sarah.lewis@nottingham.ac.uk

'Division of Epidemiology and Public Health, Faculty of Medicine and Health Sciences, University of Nottingham, Clinical Sciences Building 2, Nottingham City Hospital, Hucknall Road, Nottingham NG5 1 PB, UK

Full list of author information is available at the end of the article
}

the most prevalent modifiable risk factors for adverse pregnancy outcomes $[5,6]$.

In many developed countries, the rates of smoking in pregnancy have been declining over recent decades [7] with current prevalence estimates between 10\% - 19\% [8], and data from the UK suggesting that one out of eight women smoke throughout the pregnancy [9]. Moreover, this decline has not been consistent across all social classes; lower rates of decline have been noted across less advantaged socioeconomic groups [10]. Smoking in pregnancy in developed countries tends to be higher among women who have low income and poor education [11].

Stillbirth rates widely vary across low, middle and high-income countries [12-14]. The lowest rates have been observed in Finland with 2.0 per 1000 live births and in the developing world rates are much higher at more than 40 per 1000 live births in countries like Nigeria and Pakistan [6]. Two previous systematic reviews and meta-analyses have estimated the size of effect 
of smoking during pregnancy and the risk of stillbirth. A report by the United States Surgeon General [15] showed a relative risk of stillbirth of 1.2-1.8 within smokers versus non-smokers. The analysis was based on three studies [16-18] conducted in two countries, the United States and Sweden. Recent stillbirth statistics by Flenady et al [8] was based on a systematic review from high-income countries; and four studies were included in the meta-analysis of the association between any smoking in pregnancy and the risk of stillbirth yielding an odds ratio of 1.36 (95\% CI 1.27, 1.46). Results from these published reviews were limited to literature from developed countries; yet stillbirth rates are much higher in developing countries, and it is therefore imperative to conduct a comprehensive review, which reflects the impact of smoking in pregnancy on stillbirth. We have conducted a systematic review and meta-analysis to provide contemporary estimates of the association between maternal smoking in pregnancy and the risk of stillbirth.

\section{Methods}

A detailed electronic search was performed through four databases namely MEDLINE, EMBASE, Psych Info and Web of Science. All relevant published studies with sufficient data on maternal cigarette or tobacco smoking during pregnancy with the outcome of stillbirth were included. Due to the nature of the research objective, there were no randomised control trials (RCTs) identified, and observational studies (such as cohort design, case control studies and cross sectional surveys) were considered using the standard guidelines of Meta-analysis of Observational Studies in Epidemiology (MOOSE) [19]. All relevant studies published in the English language, up to $31^{\text {st }}$ December 2012 were included in the review. Case reports, non-English publications and those, which only involved passive or environmental smoke information, were excluded. We carefully considered studies where the study populations were similar to avoid duplication of cases; where studies were based on exactly the same population during the same time period, but there were differences in sample sizes, we selected the results from the publication with the larger sample size for inclusion in the meta-analysis, though those excluded from quantitative analysis were included in a narrative synthesis.

\section{Search strategy}

We developed search terms based on medical subject headings $(\mathrm{MeSH})$, free text words and words in the title or abstract. We combined search terms of exposure (smoking during pregnancy) with the outcome of the study (stillbirth). The MeSH terms used included combinations of the terms: stillbirth, maternal smoking, and pregnancy, also applied with special characters (\$, *), wherever required. The study protocol was agreed within the team, however it was not published prior to commencement of the review. We also checked the reference list of all identified papers and the most recent similar reviews for any additional studies. A complete online search strategy along with search terms is attached (Additional file 1).

\section{Study selection and outcome definition}

The electronic search was performed with a three-stage approach; first titles were screened by TM and AA, then abstracts were screened by two authors independently (TM and SL). For studies, which appeared to be eligible, full texts were obtained and reviewed independently by two authors who further performed data extraction independently on pre-piloted forms, and discrepancies were resolved by consensus. We also obtained full texts for those studies where a decision could not be made based on title and abstract. Data extraction forms included followings; study design, exposure validation, definition of exposure (smoking) and outcome (stillbirth), confounders adjustments, sample size and study location.

Since stillbirth is defined differently in different countries and different studies, stillbirth for this review was defined as fetal loss or death at 20 weeks gestation and above [20]; this included both early (20-28 weeks gestation) and late (after 28 weeks) stillbirth enabling inclusion of a wider range of international studies. Sensitivity analysis was done using alternative definitions of stillbirth:

a) Stillbirth at $\geq 20$ weeks of gestation

b) Stillbirth at $\geq 24$ weeks of gestation

c) Stillbirth at $\geq 28$ weeks of gestation

Where both unadjusted and adjusted measures were reported, the latter were extracted. Quality assessment was based on a Newcastle Ottawa Scale (NOS). Cohort and case control studies were awarded up to a maximum quality score of nine and cross sectional studies were given up to a maximum score of seven [21]. A definition of 'high quality', was given to cohort and case control studies with a score of 7 or above or crosssectional studies with a score of 5 or above; the rest were deemed 'low quality' [21]. Each quality assessment (NOS) was conducted by at least by two authors independently and then findings were verified.

\section{Statistical analysis}

Meta-analysis was performed using Rev-Man 5.3 [22] with a random effects model. The $\mathrm{I}^{2}$ statistic was applied to calculate heterogeneity $\left(\mathrm{I}^{2}\right.$ more than $75 \%$ was considered high heterogeneity, more than $50 \%$ moderate and $25 \%$ was considered as low heterogeneity) [22]. The odds ratio for the overall effect of smoking in pregnancy from each study was used, which was the main outcome 
reported in included literature. The ratio was calculated from the available data, wherever feasible. Studies included in the meta-analysis were further considered for subgroup analysis based on study quality, definition of stillbirth and cigarette consumption (1-9 cig/day and $\geq 10 \mathrm{cig} /$ day). The funnel plot method was used to assess publication bias. Studies without statistical data presentation, and those where cases were potentially overlapping between publications, were considered in a narrative synthesis.

\section{Results}

The initial database search produced 2,934 papers. We found 1,168 duplicate studies; meaning the same study was obtained from more than one of the four databases used in the review. After removal of duplicates, 1,766 study titles were screened, 94 were found eligible for abstract screening and 35 papers were considered for full text analysis. Out of 35 full texts sought, 34 were obtained within the available research timelines, and of these, 29 were included in the systematic review [12-14,16-18,23-45]. Eight full text studies, which did not have sufficient qualitative or quantitative data relevant to this review, were excluded [46-53]. An additional five relevant studies [54-58] were identified from references listed in the full text eligible studies. Details of the electronic search are explained in Figure 1. Out of the 34 included studies, 24 were included in the meta-analysis [12-14,17,23,24,26-29,31-41,54-56] and the other remaining ten in the qualitative synthesis $[16,18,25,30,42-45,57,58]$. One of the studies, [23] used two separate study designs, case control and bidirectional crossover methodology; therefore the study was considered in the meta-analysis separately as two observations resulting in 25 studies included in the quantitative synthesis. Two studies references [30,31] were derived from the same dataset (Missouri, USA) conducted in the same timeframe (19781997). Study [31] with a higher sample size was included in the meta-analysis and the other [30] was considered in the narrative synthesis. Four studies $[16,17,25,29]$ used the same study population (Swedish National registry data) with differences in sample size and study methodology. Studies $[17,25]$ had the same time frame, with study [17] having a higher sample size. Studies $[16,17,29]$ had overlapping but not the exact dataset time frames. Study [16] time period (1983 - 1985) was exceeded by study 17 (1983-1989) which also had a higher sample size. Study

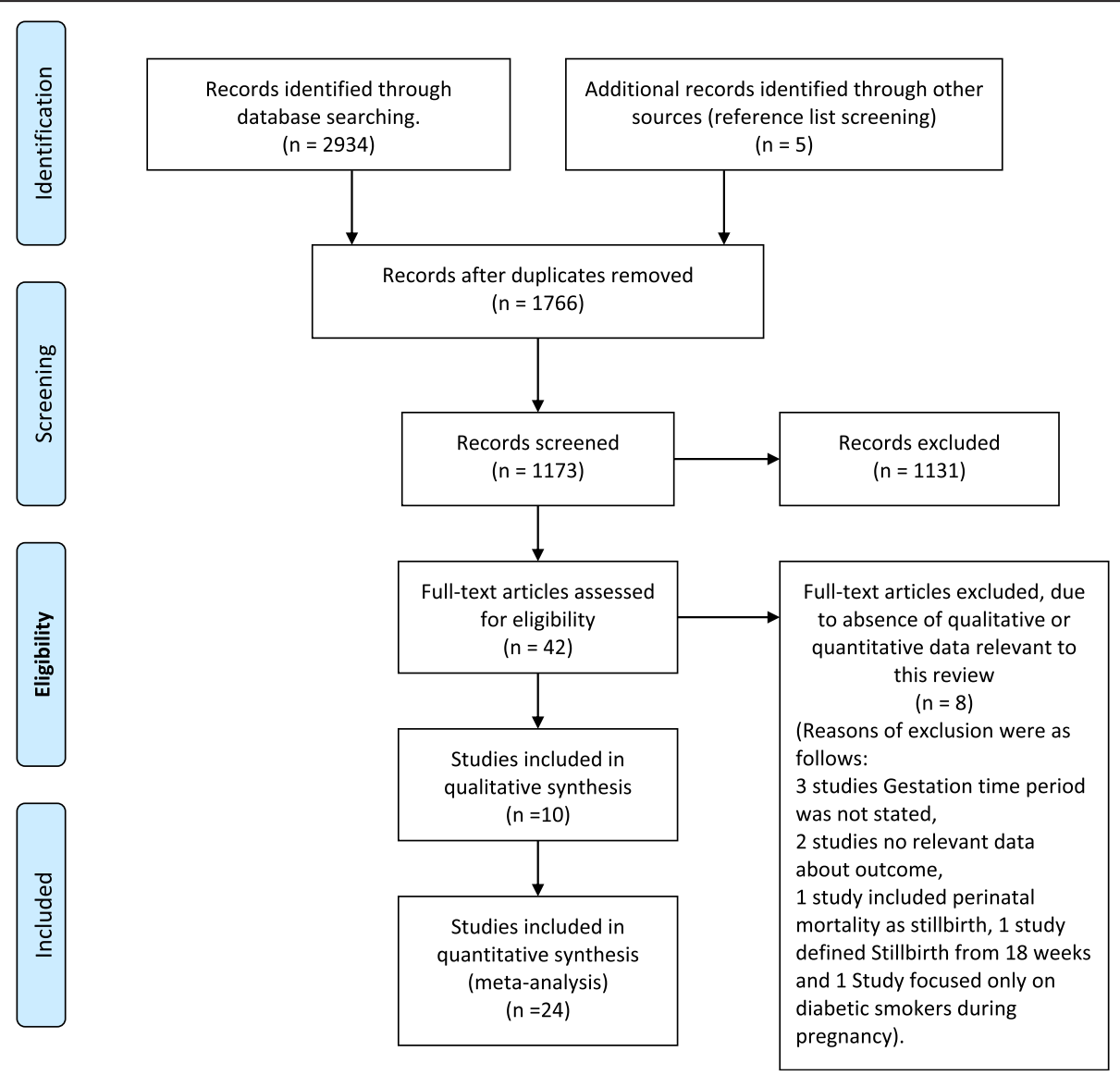

Figure 1 Flow diagram of included and excluded studies. 
[29] used the data for 1984 and 1991 for comparative purposes. To avoid having any woman's data being counted twice in the meta-analysis, only the estimates obtained for 1991 data [29] were used in the meta-analysis. Two studies $[17,29]$ were included in the meta-analysis whilst the other two studies $[16,25]$ were included in the narrative synthesis only.

Included studies are further detailed in Table 1.

Out of the 34 eligible studies (Table 1), eight were case control studies [23-28,54,55], five cross sectional $[16,18,40,41,45]$ and twenty-one were cohort studies [12-14,17,29-39,42-44,56-58]. Fourteen were conducted in Europe, [13,14,16,17,25,27,29,32,36-39,43,54], four in Australia [34,41,56,58], fourteen in North America $[12,18,23,26,28,30,31,33,35,42,44,45,55,57]$, one in Asia [40] and one in South America [24]. The largest sample size observed was $7,792,990$ [45] with the smallest being 202 [25]. Ten studies [13,16,25,26,30-32,35,54,55] did not present their results with odds ratios but had sufficient data to calculate the ratio using STATA 12, which was further used in the meta-analysis. Seven studies $[18,42-45,57,58]$ did not present estimates or sufficient data to calculate an odds ratio; therefore they were included in the narrative synthesis. Based on NOS scale, overall study quality was moderately satisfactory. Ten cohort studies [12,14,17,32,35-39,42] and four case control studies $[23,24,54,55]$ were considered of high quality with seven or more points, and only two cross sectional studies $[16,45]$ were of high quality having five or more points. Scores ranged from 2 to 9 and the median score was 6 .

\section{The type of exposure}

Eleven studies clearly defined the level of exposure (smoking) [14,16,17,25,29,31,33,34,38,54,56]. Most of the studies used categories of $1-9 \mathrm{cig} /$ day and $\geq 10 \mathrm{cig} /$ day to categorise participants according to the number of cigarettes or packs consumed daily, but the Robson study [41] used slightly different categories. Seventeen studies did not define smoking according to level of cigarette consumption [12,13,24,26-28,30,32,35,36,40,41,43,45,55,57,58] but according to the smoking status (yes/no). Fifteen studies collected exposure information during pregnancy $[12-14,16,17,27,29,30,32,33,38,42,43,54,56]$ and the rest of the studies [18,23-26,31,34,35,39-41,44,45,55,57,58] obtained the information after delivery. None of the studies reported biochemical validation of smoking status such as salivary cotinine assessments.

\section{Data collection on outcome}

The definition of stillbirth varied across studies. Twenty-one out of thirty-four studies included stillbirth from early gestational age $\geq 20$ weeks $[18,23,30,31,33,34$, $36,37,41-43,45,56-58$ ],$\geq 22$ weeks [54], $\geq 23$ weeks [35], and four studies used birth weight to estimate the gestational week of stillbirth (Birth weight $\geq 500 \mathrm{~g}$, $[24,26,28,44]$. The rest of the studies used late gestational ages as the cut off values for stillbirth, $\geq 24$ weeks in four studies $[12,13,27,32], \geq 28$ weeks in nine studies $[14,16,17,25,29,38-40,55]$. In all studies, outcome data was either obtained from medical or clinical records, medical data sets or birth registry records.

Fourteen studies included in the quantitative synthesis adjusted for one or more confounders (Table 1). Maternal age was the most common adjusted factor. Five studies adjusted for socioeconomic status (SES) including education $[13,14,37,38,40]$. Other factors that were adjusted for include parity, BMI, ethnicity/race, infant sex, perinatal care and marital status, alcohol, caffeine intake and cohabitation. Four studies [24,27,36,39] did not adjust for any confounders and six studies $[26,31,32,35,54,55]$ had OR calculated therefore considered as not adjusted for any confounders. Factors adjusted in cohort studies varied widely. Several studies $[12,17,28,34,37,41,56]$ considered one or more medical conditions such as pregnancy complications, diabetes mellitus and hypertension as possible confounders.

\section{Meta-analysis of maternal smoking and risk of stillbirth}

In meta-analysis of all 25 studies (Figure 2), smoking during pregnancy was associated with a $47 \%$ increase in the odds of stillbirth (OR 1.47, 95\% CI 1.37, 1.57, p < 0.0001) with an overall moderate heterogeneity $\left(\mathrm{I}^{2}=79 \%\right)$. There was no significant difference in the size of this estimate between study designs $(\mathrm{p}=0.11)$; the odds of stillbirth were increased by $34 \%$ in relation to smoking in pregnancy in case-control studies (OR 1.34, 95\% CI 1.23, 1.45, $\mathrm{p}<0.0001,8$ studies), $49 \%$ in cohort studies (OR 1.49, 95\% CI $1.35,1.64, \mathrm{p}<0.0001,15$ studies $)$ and $62 \%$ in crosssectional studies (OR 1.62, 95\% CI 1.31, 2.00, p $<0.0001,2$ studies). Results were more heterogeneous for cohort studies than other study types.

\section{Subgroup analysis by study quality}

Results from high quality score studies showed that women who smoke during pregnancy, are $41 \%$ more likely to have a stillbirth compared to women who do not smoke during pregnancy (OR 1.41, 95\% CI 1.28, $1.55, \mathrm{p}<0.0001,14$ studies). Low quality score studies showed that women who smoke during pregnancy were at $49 \%$ increased odds of stillbirth compared to women who do not smoke during pregnancy (OR 1.49, 95\% CI $1.33,1.67, \mathrm{p}<0.0001,11$ studies). There was no significant difference between the two subgroups $(\mathrm{p}=0.44)$.

\section{Subgroup analysis by categories of cigarette consumption}

Seven studies $(14,26 \mathrm{a}-\mathrm{b}, 31,34,45,48)$ were included in this subgroup analysis, with consumption categorised as $1-9 \mathrm{cig} /$ day and $\geq 10 \mathrm{cig} /$ day (Figure 3). One study [29] 
Table 1 Table of included studies

\begin{tabular}{|c|c|c|c|c|c|c|c|c|}
\hline Reference & $\begin{array}{l}\text { Author } \\
\text { year }\end{array}$ & $\begin{array}{l}\text { Study } \\
\text { type }\end{array}$ & Population & Location & $\begin{array}{l}\text { Exposure: } \\
\text { smoking during } \\
\text { pregnancy and } \\
\text { validity }\end{array}$ & $\begin{array}{l}\text { Definition of stillbirth } \\
\text { (outcome) and } \\
\text { gestational period }\end{array}$ & Estimates & $\begin{array}{l}\text { Factors adjusted for in individual } \\
\text { studies if available }\end{array}$ \\
\hline \multicolumn{9}{|c|}{ Studies included in meta-analysis } \\
\hline \multirow[t]{2}{*}{12} & \multirow{2}{*}{$\begin{array}{l}\text { Wood et al } \\
\text { (2009) }\end{array}$} & \multirow[t]{2}{*}{ Cohort } & \multirow[t]{2}{*}{158,502} & \multirow{2}{*}{$\begin{array}{l}\text { Alberta Perinatal Health } \\
\text { Program, 1991-2004 } \\
\text { Canada }\end{array}$} & Smokers: Yes & \multirow{2}{*}{$\begin{array}{l}\text { Fetal death after at least } \\
24 \text { completed weeks of } \\
\text { gestation. }\end{array}$} & \multirow[t]{2}{*}{ Adjusted OR 1.04 (0.74-1.47) } & \multirow{2}{*}{$\begin{array}{l}\text { Maternal age, diabetes, hypertension } \\
\text { previous caesarean section. }\end{array}$} \\
\hline & & & & & Not validated & & & \\
\hline \multirow[t]{2}{*}{13} & \multirow{2}{*}{$\begin{array}{l}\text { Smith et al } \\
(2007)\end{array}$} & \multirow[t]{2}{*}{ Cohort } & \multirow[t]{2}{*}{84,769} & \multirow{2}{*}{$\begin{array}{l}\text { Scotland morbidity } \\
\text { records 1991-2001, UK }\end{array}$} & Smokers: Yes & \multirow{2}{*}{$\begin{array}{l}\text { Fetal death after at least } 24 \\
\text { completed weeks of } \\
\text { gestation. }\end{array}$} & \multirow{2}{*}{$\begin{array}{l}\text { OR for smoking was calculated } \\
2.15(95 \% \text { Cl 1.74-2.65) }\end{array}$} & \multirow{2}{*}{$\begin{array}{l}\text { Age, deprivation, height, BMI, Marital } \\
\text { Status. }\end{array}$} \\
\hline & & & & & Not validated & & & \\
\hline \multirow[t]{3}{*}{14} & \multirow{3}{*}{$\begin{array}{l}\text { Hogberg } \\
\text { and } \\
\text { Cnattingius } \\
\text { (2007) }\end{array}$} & \multirow[t]{3}{*}{ Cohort } & \multirow[t]{3}{*}{526,691} & \multirow{3}{*}{$\begin{array}{l}\text { Sweden - Swedish } \\
\text { National study 1983- } \\
\text { 2001- Participants with } \\
\text { two consecutive } \\
\text { pregnancies. }\end{array}$} & Smokers: Yes & \multirow{3}{*}{$\begin{array}{l}\text { Fetal death after at least } 28 \\
\text { completed weeks of } \\
\text { gestation. }\end{array}$} & \multirow{3}{*}{$\begin{array}{l}\text { OR for smoking in both Pregnancies } \\
1.35 \text { (1.15-1.58). 1-9cig/day OR } 1.16 \\
(0.92-1.46) \geq 10 \text { cig/day OR } 1.55 \\
\text { (1.17-2.04). OR for smoking in the } \\
1^{\text {st }} \text { pregnancy only OR } 1.02(0.79- \\
1.30), 1-9 \text { cig/day OR } 1.13(0.9-1.39) \text {, } \\
\geq 10 \text { cig/day OR } 1.14 \text { (0.87-1.51). OR } \\
\text { for smoking in } 2^{\text {nd }} \text { pregnancy only } \\
0.84(0.55-1.26), 1-9 \text { cig/day OR } 1.10 \\
(0.89-1.35), \geq 10 \text { cig/day OR } 1.45 \\
(1.16-1.81) .\end{array}$} & \multirow{3}{*}{$\begin{array}{l}\text { Maternal age, education, cohabiting } \\
\text { with infant's father, mother's country } \\
\text { of birth, year of second delivery, } \\
\text { inter-pregnancy interval and stillbirth } \\
\text { in first pregnancy. }\end{array}$} \\
\hline & & & & & $\begin{array}{l}1-9 \text { cig/day, } \geq 10 \\
\text { cig/day. }\end{array}$ & & & \\
\hline & & & & & Not validated & & & \\
\hline \multirow[t]{5}{*}{26} & \multirow{5}{*}{$\begin{array}{l}\text { Salihu et al } \\
\text { (2008) }\end{array}$} & \multirow{5}{*}{$\begin{array}{l}\text { Case } \\
\text { control }\end{array}$} & \multirow[t]{5}{*}{$1,444,378$} & \multirow{5}{*}{$\begin{array}{l}\text { USA - Missouri 1978- } \\
1997 .\end{array}$} & Smokers: Yes & \multirow{5}{*}{$\begin{array}{l}\text { In-utero fetal death at least } \\
20 \text { week into gestation. }\end{array}$} & \multirow{2}{*}{$\begin{array}{l}\text { a) Bidirectional case cross-over } \\
\text { OR } 1.20(1.03-1.39) 1-9 \mathrm{cig} / \text { day OR } \\
1.16(0.88-1.53) 10-19 \mathrm{cig} / \text { day OR } \\
1.10(0.90-1.24) \geq 20 \mathrm{cig} / \text { day OR } \\
1.21(1.01-1.50),\end{array}$} & \multirow[t]{5}{*}{ Maternal race and age. } \\
\hline & & & & & 1-9 cig/day, & & & \\
\hline & & & & & 10-19 cig/day, & & b) Case Control OR 1.34 (1.26-1.43) & \\
\hline & & & & & $\geq 20$ cig/day. & & $\begin{array}{l}1-9 \text { cig/day OR 0.94 (0.76-1.70) } \\
10-19 \text { cig/day OR } 1.31(1.22-1.41)\end{array}$ & \\
\hline & & & & & Not validated & & $\geq 20 \mathrm{cig} /$ day OR 1.43 (1.31-1.54). & \\
\hline \multirow[t]{7}{*}{$34^{* * *}$} & $\begin{array}{l}\text { Ahlenius } \\
\text { and }\end{array}$ & Cohort & $\begin{array}{l}94,270 \text { in } \\
1984 \text { and }\end{array}$ & $\begin{array}{l}\text { Sweden - Using the } \\
\text { Swedish medical birth }\end{array}$ & Smokers: Yes. & $\begin{array}{l}\text { Death of a fetus before or } \\
\text { during delivery with a }\end{array}$ & $\begin{array}{l}\text { Combined (1984 and 1991) OR } \\
1.37 \text { (1.17 to } 1.61)\end{array}$ & Maternal age and parity. \\
\hline & $\begin{array}{l}\text { Thomassen } \\
\text { (1999) }\end{array}$ & & $\begin{array}{l}124,201 \text { in } \\
1991 .\end{array}$ & $\begin{array}{l}\text { register in } 1984 \text { and } \\
1991\end{array}$ & $1-9$ cig/day, $\geq 10$ & $\begin{array}{l}\text { gestational duration of at } \\
\text { least } 28 \text { weeks. }\end{array}$ & 1-9 Cig/day OR 1.22 & \\
\hline & & & & & cig/day. & & $(1.00-1.48)$ & \\
\hline & & & & & Not validated & & $\geq 10$ Cig/day OR 1.62 (1.31-2.00) & \\
\hline & & & & & & & 1991 OR & \\
\hline & & & & & & & 1.47 (1.19 to 1.82$)$ & \\
\hline & & & & & & & >10 Cig/day OR 1.97 (1.51-2.59) & \\
\hline
\end{tabular}


Table 1 Table of included studies (Continued)

\begin{tabular}{|c|c|c|c|c|c|c|c|c|}
\hline \multirow[t]{3}{*}{$36^{* * *}$} & \multirow{3}{*}{$\begin{array}{l}\text { Aliyu et al } \\
\text { (2007) }\end{array}$} & \multirow[t]{3}{*}{ Cohort } & \multirow[t]{3}{*}{$1,436,725$} & \multirow{3}{*}{$\begin{array}{l}\text { USA Missouri - Using } \\
\text { Missouri maternally } \\
\text { linked cohort data } \\
1978-1997\end{array}$} & Smokers: Yes & \multirow{3}{*}{$\begin{array}{l}\text { In utero fetal death } \\
\text { at }<=20 \text { weeks gestation }\end{array}$} & \multirow{3}{*}{$\begin{array}{l}\text { OR(originally presented as HRs) } \\
1.43(1.36-1.51)\end{array}$} & \multirow[t]{3}{*}{$N / A^{*}$} \\
\hline & & & & & $\begin{array}{l}\text { 1-9 cig/day, } \\
10-19 \mathrm{cig} / \mathrm{day} \\
\geq 20 \mathrm{cig} / \mathrm{day}\end{array}$ & & & \\
\hline & & & & & Not validated & & & \\
\hline \multirow[t]{2}{*}{37} & \multirow{2}{*}{$\begin{array}{l}\text { Gray et al } \\
\text { (2009) }\end{array}$} & \multirow[t]{2}{*}{ Cohort } & \multirow[t]{2}{*}{532,016} & \multirow{2}{*}{$\begin{array}{l}\text { UK- Scotland, National } \\
\text { study 1994-2003. }\end{array}$} & Smokers: Yes. & \multirow[t]{2}{*}{ 24-44 weeks' gestation } & \multirow[t]{2}{*}{ OR $1.75(1.61-1.91)$} & \multirow[t]{2}{*}{$N / A^{*}$} \\
\hline & & & & & Not validated & & & \\
\hline \multirow[t]{2}{*}{38} & \multirow[t]{2}{*}{$\begin{array}{l}\text { McCowan } \\
\text { et al (2007) }\end{array}$} & \multirow[t]{2}{*}{ Cohort } & \multirow[t]{2}{*}{69,173} & $\begin{array}{l}\text { New Zealand - National } \\
\text { Women's }\end{array}$ & Smokers: Yes & $\begin{array}{l}\text { Birth of a baby with no } \\
\text { signs of life delivered at } \geq\end{array}$ & \multirow[t]{2}{*}{ OR $1.33(0.99-1.79)$} & \multirow{2}{*}{$\begin{array}{l}\text { Infant sex, maternal ethnicity, parity, } \\
\text { marital status, maternal age at } \\
\text { delivery, history of previous } \\
\text { miscarriage, history of previous } \\
\text { abortion, previous low-birth weight } \\
\text { infant, previous C-section. }\end{array}$} \\
\hline & & & & $\begin{array}{l}\text { Hospital, Auckland, New } \\
\text { Zealand from } 1993 \text { to } \\
2000\end{array}$ & Not validated & $\begin{array}{l}20 \text { weeks or with a birth } \\
\text { weight of }>400 \mathrm{~g} \text { if } \\
\text { gestation was not known. }\end{array}$ & & \\
\hline \multirow[t]{2}{*}{39} & \multirow{2}{*}{$\begin{array}{l}\text { Miller et al } \\
(2010)\end{array}$} & \multirow[t]{2}{*}{ Cohort } & \multirow[t]{2}{*}{359,747} & \multirow{2}{*}{$\begin{array}{l}\text { Canada - Ontario state } \\
\text { 2004-2006 }\end{array}$} & Smokers: Yes & \multirow{2}{*}{$\begin{array}{l}\text { Death of fetus at } 20 \text { weeks } \\
\text { of gestation }\end{array}$} & \multirow[t]{2}{*}{ Overall OR 1.58 (1.38-1.81) } & \multirow[t]{2}{*}{ Age, parity, multiple gestation } \\
\hline & & & & & Not validated & & & \\
\hline \multirow[t]{2}{*}{40} & \multirow{2}{*}{$\begin{array}{l}\text { Moshin et } \\
\text { al (2005) }\end{array}$} & Cohort & 433,227 & Australia - New South & Smokers: Yes & Fetal death at $\geq 20$ weeks & Overall adjusted OR 1.17 & Maternal age, maternal country of \\
\hline & & & & Wales 1998-2002 & Not validated & $\begin{array}{l}\text { gestation or of } 400 \mathrm{~g} \text { or } \\
\text { more birth weight. }\end{array}$ & OR $1.05(0.94-1.17)$ & $\begin{array}{l}\text { diabetes mellitus, and first antenatal } \\
\text { care visit. }\end{array}$ \\
\hline $17^{* * *}$ & Raymond & Cohort & 638,242 & Sweden National study & Smokers: Yes & Fetus death at 28 weeks of & OR $1.4(1.2-1.4)$ & Age, parity, pregnancy complications, \\
\hline & et al (1994) & & & 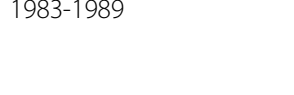 & Not validated & gestation or more. & & $\begin{array}{l}\text { hypertensive diseases, diabetes, placental } \\
\text { complications and intrauterine growth } \\
\text { retardation (IUGR). }\end{array}$ \\
\hline 42 & Reddy et al & Cohort & 174,809 & USA - 12 clinic centres & Smokers: Yes & Fetal death at 23 weeks & OR (originally presented as HRs) & $N / A^{*}$ \\
\hline & (2006) & & & $\begin{array}{l}\text { and } 19 \text { hospital sites. } \\
\text { 2002-2008. }\end{array}$ & Not validated & gestation and beyond. & & \\
\hline 43 & Sutan et al & Cohort & 541,811 & UK-Scotland, National & Smokers: Yes & Late fetal death from & OR $1.64(1.46-1.84)$ & Non stated \\
\hline & & & & study (NHS Hospital) & Not validated & 20 weeks gestation. & & \\
\hline 44 & Tuthill et al & Cohort & 16,047 & Wales - All Wales & Smokers: Yes & Mortality from 20 & OR $1.72(1.38-2.13)$ & Social class, infection, placental \\
\hline & & & & $\begin{array}{l}\text { Perinatal Survey } \\
\text { 1993-1995 }\end{array}$ & Not validated & $\begin{array}{l}\text { completed weeks of } \\
\text { gestation }\end{array}$ & & $\begin{array}{l}\text { abruption, sudden infant death } \\
\text { syndrome. }\end{array}$ \\
\hline 45 & Wisborg et & Cohort & 25,102 & Denmark - Aarhust & Smokers: Yes & Delivery of a dead fetus & OR 1.9 (1.3-2.9), & Sex of child, parity, maternal age, \\
\hline & & & & $\begin{array}{l}\text { University Hospital } \\
\text { 1989-1996. }\end{array}$ & $\begin{array}{l}1-9 \text { cig/day, } \geq 10 \\
\text { cig/day. }\end{array}$ & $\begin{array}{l}\text { occurring at } 28 \text { weeks or } \\
\text { more gestation. }\end{array}$ & $\begin{array}{l}\text { 1-9 cig/day OR } 1.5(0.9-2.4) \geq 10 \\
\text { cig/day } 1.8(1.2-2.8)\end{array}$ & \\
\hline & & & & & Not validated & & & \\
\hline 46 & Winbo et al & Cohort & $1,412,747$ & Sweden - National & Smokers: Yes & Death of fetus after & OR stillbirths $\geq 37$ weeks & Non stated \\
\hline & & & & & Not validated & $\begin{array}{l}\text { competing } 28 \text { weeks of } \\
\text { gestation. }\end{array}$ & $\begin{array}{l}\text { compared with live births } 1.08 \\
(0.96-1.22)\end{array}$ & \\
\hline 27 & Ferraz and & Case & 11,483 & Brazil - Natal city and & Smokers: Yes & Stillborn cases weighing & OR 1.4 (1.0-2.0) & Non stated \\
\hline & & & & $\begin{array}{l}\text { Nate of RIo Grande do } \\
\text { Norte 1984-1986 }\end{array}$ & Not validated & & & \\
\hline
\end{tabular}


Table 1 Table of included studies (Continued)

\begin{tabular}{|c|c|c|c|c|c|c|c|c|}
\hline \multirow[t]{2}{*}{29} & \multirow{2}{*}{$\begin{array}{l}\text { Dodds et al } \\
\text { (2006) }\end{array}$} & \multirow{2}{*}{$\begin{array}{l}\text { Case } \\
\text { control }\end{array}$} & \multirow[t]{2}{*}{494} & \multirow{2}{*}{$\begin{array}{l}\text { Canada - Nova Scotia } \\
\text { and Eastern Ontario } \\
\text { 1999-2001 }\end{array}$} & Smokers: Yes & \multirow{2}{*}{$\begin{array}{l}\text { Not specified - Death } \\
\text { before delivery of a fetus } \\
\text { weighing } 500 \mathrm{~g} \text { or more }\end{array}$} & \multirow{2}{*}{$\begin{array}{l}\text { OR (originally presented in HRs) } \\
\text { Smoking during } 1^{\text {st }} \text { trimester } 2.13 \\
(1.23-3.64)\end{array}$} & \multirow[t]{2}{*}{$N / A^{*}$} \\
\hline & & & & & Not validated & & & \\
\hline \multirow[t]{2}{*}{30} & \multirow{2}{*}{$\begin{array}{l}\text { Efkarpidis et } \\
\text { al (2004) }\end{array}$} & \multirow{2}{*}{$\begin{array}{l}\text { Case } \\
\text { control }\end{array}$} & \multirow[t]{2}{*}{599} & \multirow{2}{*}{$\begin{array}{l}\text { UK - Nottingham City } \\
\text { Hospital 1991-1997. }\end{array}$} & Smokers: Yes & \multirow{2}{*}{$\begin{array}{l}\text { Definition of stillbirth not } \\
\text { stated- pregnancies } \\
\text { considered from } \geq 24 \text { weeks }\end{array}$} & \multirow[t]{2}{*}{ OR 1.2 (0.64-1.6) } & \multirow[t]{2}{*}{ Non stated } \\
\hline & & & & & Not validated & & & \\
\hline \multirow[t]{3}{*}{31} & \multirow{3}{*}{$\begin{array}{l}\text { Froen et al } \\
\text { (2002) }\end{array}$} & \multirow{3}{*}{$\begin{array}{l}\text { Case } \\
\text { control }\end{array}$} & \multirow[t]{3}{*}{598} & \multirow{3}{*}{$\begin{array}{l}\text { Norway - Oslo } \\
\text { 1986-1995. }\end{array}$} & Smokers: Yes & \multirow{3}{*}{$\begin{array}{l}\text { Intrauterine death before } \\
\text { onset of labour of a } \\
\text { fetus }>=22 \text { weeks gestation } \\
\text { or }>=500 \mathrm{~g} \text { body mass }\end{array}$} & \multirow{3}{*}{$\begin{array}{l}\text { OR calculated Using Stats } 1.81 \\
(1.02-3.15) 1-9 \text { cig/day OR } 1.0 \\
(0.43-2.12), \geq 10 \text { cig/day OR } 3.04 \\
(1.13-8.19)\end{array}$} & \multirow[t]{3}{*}{$N / A^{*}$} \\
\hline & & & & & $\begin{array}{l}1-9 \text { cig/day, } \\
\geq 10 \text { cig/day. }\end{array}$ & & & \\
\hline & & & & & Not validated & & & \\
\hline \multirow[t]{2}{*}{32} & \multirow{2}{*}{$\begin{array}{l}\text { Goy et al } \\
(2008)\end{array}$} & \multirow{2}{*}{$\begin{array}{l}\text { Case } \\
\text { control }\end{array}$} & \multirow[t]{2}{*}{510} & \multirow{2}{*}{$\begin{array}{l}\text { Canada - Nova Scotia } \\
\text { and Eastern Ontario } \\
\text { 1999-2001. }\end{array}$} & Smokers: Yes & \multirow{2}{*}{$\begin{array}{l}\text { Not specified - Death } \\
\text { before delivery of a fetus } \\
\text { weighing } 500 \mathrm{~g} \text { or more }\end{array}$} & \multirow[t]{2}{*}{ OR $2.06(1.22-3.47)$} & Province of origin, age, inactivity \\
\hline & & & & & Not validated & & & $\begin{array}{l}\text { previous pregnancy, used fertility } \\
\text { treatment }\end{array}$ \\
\hline 33 & $\begin{array}{l}\text { Little and } \\
\text { Weiberg }\end{array}$ & $\begin{array}{l}\text { Case } \\
\text { control }\end{array}$ & 4667 & USA & Smokers: Yes & $\begin{array}{l}\text { Fetal death at } 28 \text { weeks } \\
\text { or more gestation }\end{array}$ & $\begin{array}{l}\text { OR calculated using Stata } 1.29 \\
(1.09-1.53)\end{array}$ & $N / A^{*}$ \\
\hline & & & & & 1-19 cig/day, & & OR 1-19 cig/day 1.3 (1.09-1.57), & \\
\hline & & & & & 20-29 cig/day, & & $\begin{array}{l}20-29 \mathrm{clg} / \text { day OR } 1.39(1.06-1.82) \\
\geq 30 \mathrm{cig} / \mathrm{day} \text { OR } 0.67(0.41-1.12)\end{array}$ & \\
\hline & & & & & $\geq 30 \mathrm{cig} /$ day. & & & \\
\hline & & & & & Not validated & & & \\
\hline 47 & Mishra et al & Cross- & 16,802 & India - Survey & Smokers: Yes & Delivery of a dead baby & OR $1.23(0.92-1.64)$ & Cooking smoke, anaemia, BMI, \\
\hline & (2005) & sectional & & conducted 98-99 & Not validated & at > 28 weeks of pregnancy & Unadjusted OR 1.60 (1.23-2.08). & $\begin{array}{l}\text { education, standard of living, house } \\
\text { type. }\end{array}$ \\
\hline 48 & Robson et & Cross- & 21,880 & Australia - New South & Smokers: Yes & Not stated & OR 1.65 (1.15-2.38) & Age, parity, medical/obstetric \\
\hline & & & & & $\begin{array}{l}1-10 \mathrm{cig} / \text { day } \\
>10 \mathrm{cig} / \text { day. }\end{array}$ & 20 weeks & $\begin{array}{l}\text { 1- } 10 \text { cig/day OR } 1.16(0.80-1.69) \\
>10 \text { cig/day OR } 1.68 \text { (1.17-2.41). }\end{array}$ & complications \\
\hline & & & & & Not validated & & & \\
\hline Studie & luded in narra & ative synth & lesis & & & & & \\
\hline $16^{* * *}$ & $\begin{array}{l}\text { Cnattingius } \\
\text { et al (1988) }\end{array}$ & $\begin{array}{l}\text { Cross- } \\
\text { sectional }\end{array}$ & 262,582 & $\begin{array}{l}\text { Sweden National } \\
\text { Study - Using Swedish }\end{array}$ & Smokers: Yes & $\begin{array}{l}\text { Late fetal death - still birth } \\
\text { occurring at } 28 \text { weeks of }\end{array}$ & $\begin{array}{l}\text { OR (originally presented as RR) } \\
1.37(1.20-1.57)\end{array}$ & $N / A^{*}$ \\
\hline & & & & $\begin{array}{l}\text { medical birth registry } \\
\text { covering more than } 99 \%\end{array}$ & 1-9 cig/day, & gestatıon or later & 1-9 cig/day 1.32 (1.12-1.55) & \\
\hline & & & & of all births in Sweden. & $\geq 10 \mathrm{cig} / \mathrm{day}$. & & $\geq 10$ cig/day 1.45 (1.21-1.75) & \\
\hline & & & & & Not validated & & & \\
\hline $28^{* * *}$ & $\begin{array}{l}\text { Walles } \\
\text { (1994) }\end{array}$ & $\begin{array}{l}\text { Case } \\
\text { control }\end{array}$ & 202 & $\begin{array}{l}\text { Sweden - } 5 \text { centres } \\
\text { (Boras, Helsingborg, }\end{array}$ & Smokers: Yes & $\begin{array}{l}\text { Definition of stillbirth not } \\
\text { stated, pregnancies }\end{array}$ & $\begin{array}{l}\text { OR calculated using Stata. OR } 1.18 \\
(0.62-2.26)\end{array}$ & $N / A^{*}$ \\
\hline & & & & $\begin{array}{l}\text { Karlskrona, } \\
\text { Kristianstastad and }\end{array}$ & 1-10 cig/day, & & 1-10 cig/day OR 1.33 (0.58-3.09) & \\
\hline & & & & Lund) 1983-1989. & $\geq 10 \mathrm{cig} /$ day. & $\geq 28$ weeks & 10 cig/day 1.04 (0.43-2.50) & \\
\hline & & & & & Not validated & & & \\
\hline
\end{tabular}


Table 1 Table of included studies (Continued)

\begin{tabular}{|c|c|c|c|c|c|c|c|c|}
\hline \multirow[t]{3}{*}{$35^{* * *}$} & \multirow[t]{3}{*}{$\begin{array}{l}\text { Aliyu et al } \\
\text { (2008) }\end{array}$} & \multirow[t]{3}{*}{ Cohort } & \multirow[t]{3}{*}{$1,436,628$} & \multirow{3}{*}{$\begin{array}{l}\text { USA Missouri - Using } \\
\text { Missouri maternally } \\
\text { linked cohort data } \\
1978-1997 .\end{array}$} & Smokers: Yes. & \multirow[t]{3}{*}{$\begin{array}{l}\text { In utero fetal death at }<= \\
20 \text { weeks gestation. }\end{array}$} & $\begin{array}{l}\text { OR (originally presented as HRs) } \\
1.48 \text { (1.40-1.56) }\end{array}$ & \multirow[t]{3}{*}{$N / A^{* *}$} \\
\hline & & & & & \multirow{2}{*}{$\begin{array}{l}<35 \text { years old, } \\
\geq 35 \text { yers old. } \\
\text { Not validated }\end{array}$} & & $<35$ years smokers1.43 (1.35-1.51) & \\
\hline & & & & & & & $\begin{array}{l}\geq 35 \text { years Smokers OR } \\
2.4(2.06-2.80)\end{array}$ & \\
\hline \multirow[t]{4}{*}{49} & \multirow{4}{*}{$\begin{array}{l}\text { Kleinman et } \\
\text { al (1988) }\end{array}$} & \multirow{4}{*}{ Cohort } & \multirow[t]{4}{*}{362,261} & \multirow[t]{4}{*}{ USA Missouri 1978-1983 } & Smokers: Yes & \multirow{4}{*}{$\begin{array}{l}\text { (Fetal mortality) Fetal death } \\
\text { at } 20 \text { weeks or more. }\end{array}$} & OR Primiparas & \multirow[t]{4}{*}{$N / A^{* *}$} \\
\hline & & & & & $\begin{array}{l}<1 \text { pack/day } \\
\geq 1 \text { pack/day. }\end{array}$ & & $\begin{array}{l}<1 \mathrm{pack} / \text { day } 1.36(1.16-1.59) \\
\geq 1 \mathrm{pack} / \mathrm{day} 1.62(1.34-1.97)\end{array}$ & \\
\hline & & & & & \multirow[t]{2}{*}{ Not validated } & & Multiparas & \\
\hline & & & & & & & $\begin{array}{l}<1 \text { pack/day } 1.21(1.06-1.38) \\
\geq 1 \text { pack/day } 1.15(0.99-1.34)\end{array}$ & \\
\hline \multirow[t]{2}{*}{50} & \multirow{2}{*}{$\begin{array}{l}\text { Raatikainen } \\
\text { et al (2006) }\end{array}$} & \multirow[t]{2}{*}{ Cohort } & \multirow[t]{2}{*}{25,591} & \multirow{2}{*}{$\begin{array}{l}\text { Finland-Kuopio University } \\
\text { Hospital 1989-2001. }\end{array}$} & Smokers: Yes. & $\begin{array}{l}\text { Fetal death was defined as } \\
\text { intrauterine death before }\end{array}$ & \multirow[t]{2}{*}{ No estimates } & \multirow[t]{2}{*}{$N / A^{* *}$} \\
\hline & & & & & Not validated & $\begin{array}{l}22 \text { weeks of pregnancy or } \\
500 \mathrm{~g} \text { fetal weight. }\end{array}$ & & \\
\hline \multirow[t]{3}{*}{51} & \multirow{3}{*}{$\begin{array}{l}\text { Aliyu et al } \\
\text { (2010) }\end{array}$} & \multirow[t]{3}{*}{ Cohort } & \multirow[t]{3}{*}{633,849} & \multirow{3}{*}{$\begin{array}{l}\text { US Missouri - Using } \\
\text { Missouri maternally- } \\
\text { linked cohort data files } \\
1978-1997 .\end{array}$} & Smokers: Yes & \multirow{3}{*}{$\begin{array}{l}\text { In utero fetal death at }>= \\
20 \text { weeks gestation. }\end{array}$} & Results HRs, & \multirow[t]{3}{*}{$N / A^{* *}$} \\
\hline & & & & & \multirow[t]{2}{*}{ Not validated } & & $\begin{array}{l}<15 \text { years } 3.3(1.4-7.8), 15-19 \text { years } \\
1.7(1.5-1.9), 20-24 \text { years } \\
1.5(1.4-1.6)\end{array}$ & \\
\hline & & & & & & & $\begin{array}{l}\text { No sufficient data to calculate } \\
\text { Odds ratios. }\end{array}$ & \\
\hline \multirow[t]{2}{*}{52} & \multirow{2}{*}{$\begin{array}{l}\text { Bai et al } \\
(2000)\end{array}$} & \multirow[t]{2}{*}{ Cohort } & 7138 & Australia- Liverpool & Smokers: Yes & Not Clear (births occurring & Results presented in Percentages. & $N / A^{* *}$ \\
\hline & & & & 1996 to June 1998 & Not validated & $\begin{array}{l}\text { gestation age exclusion } \\
\text { criteria). }\end{array}$ & $\begin{array}{l}\text { data to calculate OR. } \\
\text {. }\end{array}$ & \\
\hline 53 & Huang et al & Cohort & 84,294 & Canada - The Royal & Smokers: Yes & Fetal deaths occurring & No estimates & $N / A^{* *}$ \\
\hline & & & & $\begin{array}{l}\text { Victoria Hospital in } \\
\text { Montreal 1961-1974 } \\
\text { and 1978-1996. }\end{array}$ & Not validated & $\begin{array}{l}\text { evidence of significant fetal, } \\
\text { maternal or placental } \\
\text { pathology weighing } 500 \mathrm{~g} \\
\text { or more. }\end{array}$ & & \\
\hline 18 & $\begin{array}{l}\text { Schramm } \\
(1997)\end{array}$ & $\begin{array}{l}\text { Cross- } \\
\text { sectional }\end{array}$ & 176,843 & $\begin{array}{l}\text { USA - Missouri } \\
1978-1990\end{array}$ & $\begin{array}{l}\text { Smokers: Yes Not } \\
\text { validated }\end{array}$ & $\begin{array}{l}\text { Fetal death greater or equal } \\
\text { to } 20 \text { weeks gestation. }\end{array}$ & $\begin{array}{l}\text { Results in RR no sufficient data to } \\
\text { calculate OR }\end{array}$ & $N / A^{* *}$ \\
\hline & & & & & & & $\begin{array}{l}\text { Smoking in } 1^{\text {st }} \text { and not in } 2^{\text {nd }} \\
\text { pregnancy RR } 1.11 \text {, Not smoking } \\
\text { in } 1^{\text {st }} \text { but in } 2^{\text {nd }} \text { pregnancy RR } \\
\text { 1.6.smoking in both pregnancies } \\
\text { RR 1.19. }\end{array}$ & \\
\hline
\end{tabular}


Table 1 Table of included studies (Continued)

\begin{tabular}{|c|c|c|c|c|c|c|c|c|}
\hline \multirow[t]{2}{*}{54} & $\begin{array}{l}\text { Salihu et al } \\
\text { (2004) }\end{array}$ & $\begin{array}{l}\text { Cross- } \\
\text { sectional }\end{array}$ & $7,792,990$ & USA - 1995-1997. & Smokers: Yes & $\begin{array}{l}\text { Intrauterine fetal demise } \\
\text { after } 20 \text { weeks of gestation. }\end{array}$ & $\begin{array}{l}\text { Estimates in Hazard ratios not } \\
\text { enough data to calculate OR. }\end{array}$ & $N / A^{* *}$ \\
\hline & & & & & Not validated & & $\begin{array}{l}\geq 40 \text { years } 2.71(1.88-3.91), 30-39 \\
\text { years } 1.58(0.96-2.60), 20-29 \text { years } \\
1.41(1.16-1.71)\end{array}$ & \\
\hline
\end{tabular}
$1.41(1.16-1.71)$

OR - Odds Ratio, HR - Hazard Ratio, RR - Risk Ratio.

$N / A^{*}$ - Original results were presented in $H R / R R$, OR was calculated using original data.

$\mathrm{N} / \mathrm{A}^{* *}$ - Studies included in descriptive synthesis only.

*** - Studies using same dataset.

Swedish National registry data set ref 16: 1933-1985, 17 and 28 : 1983-1989, ref 34: 1984 and1991.

US - Missouri data set ref 35 and 36. 


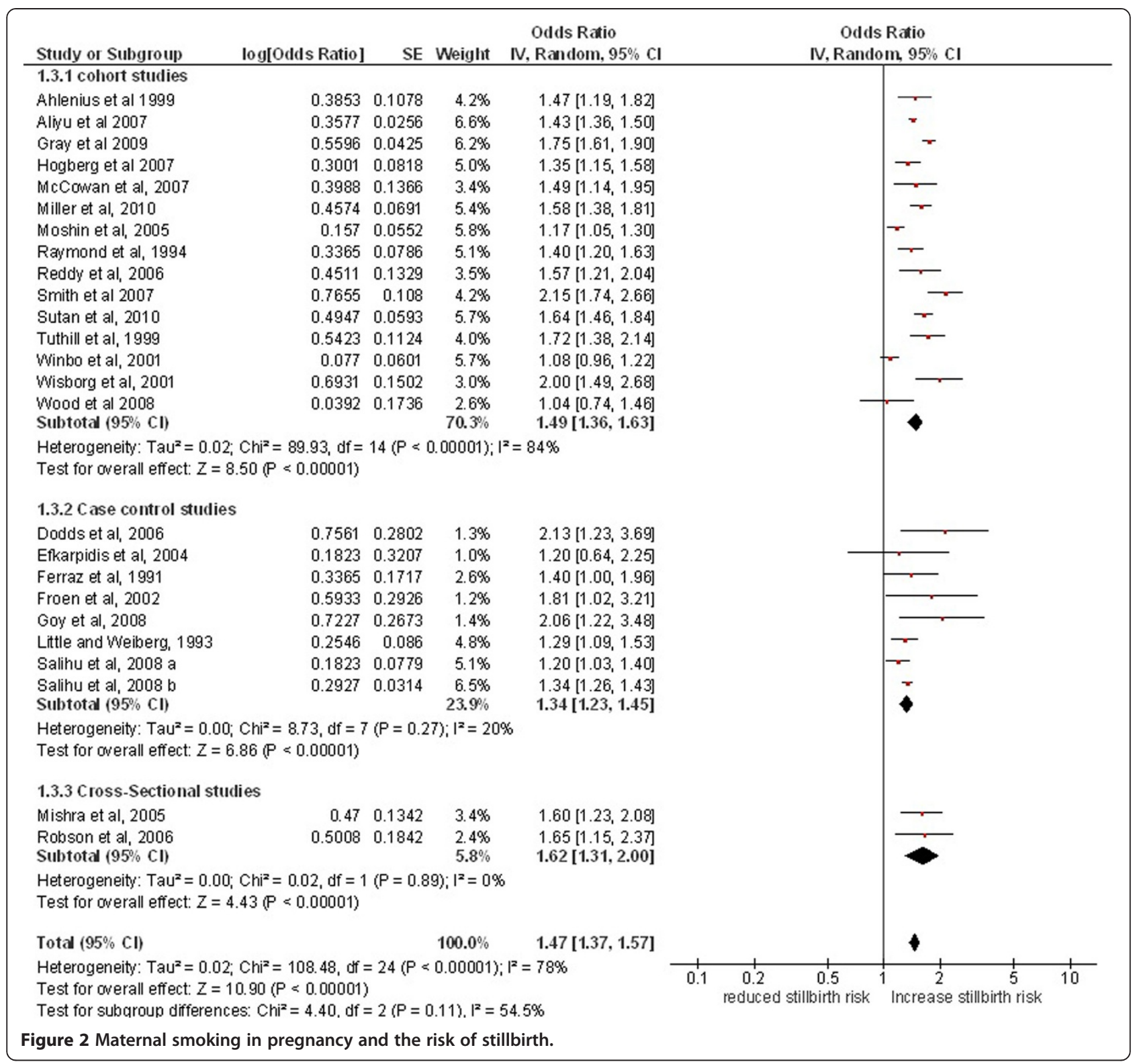

presented OR values for the $\geq 10$ cig/day category only. A study by Robson et al [41] used slightly different categories; 1-10 cig/day and >10 cig/day. This study was included in this analysis with odds ratios for the 1-10 cig/ day, and $>10 \mathrm{cig} /$ day categories used for the 1-9 cig/day and $\geq 10 \mathrm{cig} /$ day groups respectively. Further two studies by Salihu et al (2008a) [23] and Salihu et al (2008b) [23] had a classification of 1-9 cig/day, 10-19 cig/day and $\geq 20$ cig/day. There was no sufficient raw data to calculate the OR for $\geq 10 \mathrm{cig} /$ day. Therefore these studies have been included in this analysis conservatively using the ORs from the 10-19 cig/day categories (which is a lower value than the $\geq 20 \mathrm{cig} /$ day value) for the $\geq 10 \mathrm{cig} /$ day subgroup. Most of the studies included in this subgroup analysis (4 out of
7) used this categorisation (1-9 cig/day and $\geq 10 \mathrm{cig} /$ day), thus the same was applied in the subgroup analysis. Metaanalysis showed that smoking 1-9 cig/day was associated with an $9 \%$ increased odds of having a stillbirth compared to women who do not smoke in pregnancy (OR 1.09, 95\% CI $1.09,1.24, \mathrm{p}=0.55,6$ studies), whilst smoking $\geq 10 \mathrm{cig} /$ day was associated with a $52 \%$ increase in odds of stillbirth (OR 1.52, 95\% CI 1.30, 1.78, p < 0.0001, 7studies); the results for these two subgroups differed significantly $(\mathrm{p}=0.001)$.

\section{Subgroup analysis by gestation of stillbirth}

Twenty-two studies were included in this subgroup analysis (Figure 4). The meta-analysis of studies defining 


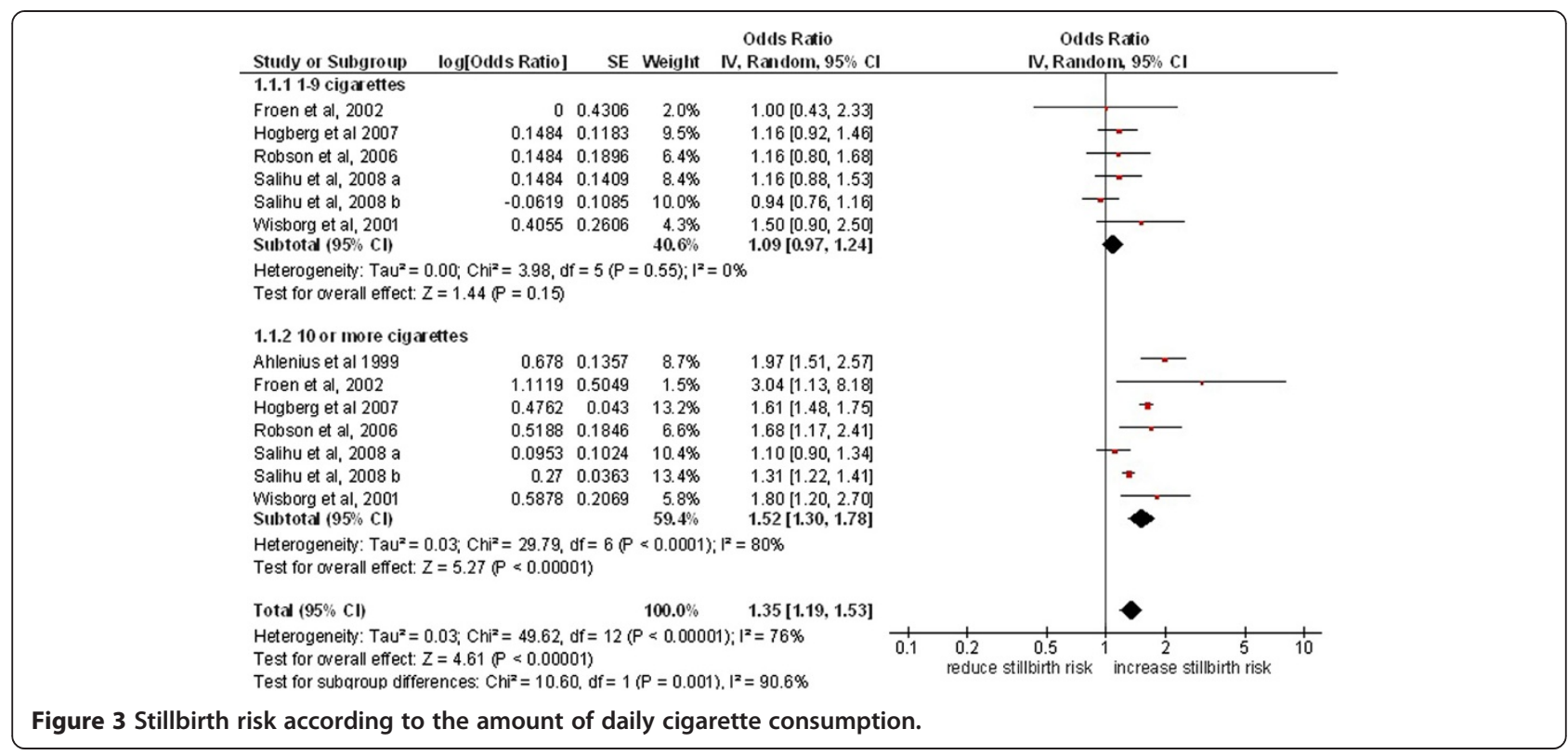

stillbirth at $\geq 20$ weeks demonstrated a $43 \%$ increase in odds for smoking mothers compared to mothers who do not smoke, (OR 1.43, 95\% CI 1.32, 1.54, p <0.0001, 11 studies), whereas studies with stillbirth defined at $\geq$ 24 weeks showed 58\% increase in odds (OR 1.58, 95\%
CI $1.21,1.2 .06, \mathrm{p}<0.0003,4$ studies) and in pooled estimates of studies with stillbirth defined at $\geq 28$ weeks, the odds was increased to $33 \%$ (OR 1.33, 95\% CI 1.18, 1.49, $\mathrm{p}<0.02,7$ studies). There was no significant difference in the odds ratios for these different subgroups $(\mathrm{p}=0.39)$.

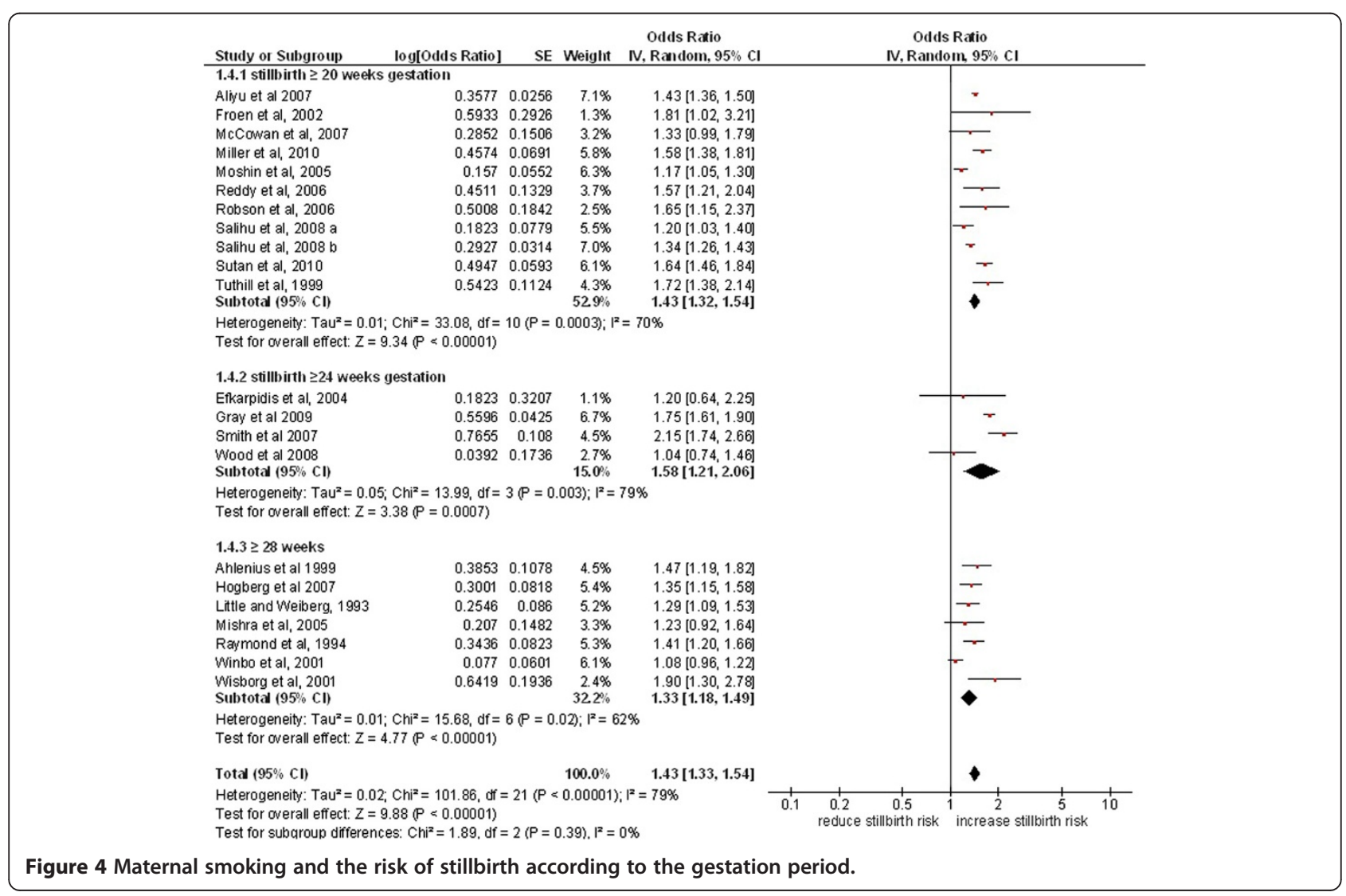




\section{Subgroup analysis by study location}

Only two studies were conducted in developing countries $[24,40]$. Both studies support evidence from research conducted in developed countries concluding that women who smoke during pregnancy are at a risk of stillbirth. A study [40] demonstrated a very marginal difference showing those women who smoke during pregnancy are at least $40 \%$ at risk of experiencing a stillbirth (OR 1.40, 95\% CI 1.0, 1.96), similar to the pooled odds ratio of studies conducted in North America (OR 1.39, 95\% CI 1.29, 1.50) $\mathrm{P}$ value $<0.0001,9$ studies) and Europe (OR 1.55, 95\% CI $1.36,1.78$, $\mathrm{P}$ value $<0.0001,11$ studies).

\section{Narrative synthesis}

Seven studies [18,42-45,57,58] presented their results in other than odds ratio and there was no sufficient data to calculate the odds ratio, and three studies $[16,25,30]$ were derived from same study population as $[17,31]$ respectively. Six of the studies [16,18,30,42,45,57] observed an association between maternal smoking in pregnancy and the risk of stillbirth but the other four $[25,43,44,58]$ observed no significant differences. Two studies $[16,42]$ observed a dose related response with high risk ratio of stillbirth in mothers who smoke $\geq 10 \mathrm{cig} /$ day compared to $1-9 \mathrm{cig} /$ day and those who smoke $\geq 1$ pack/day in contrast to $<1 \mathrm{pack} /$ day respectively. Three studies (51, 35 54) showed the risk of stillbirth to be higher in young mothers $<15$ years old (HR 3.3, 95\% CI 1.4, 7.8) and also in older mothers ( $\geq 35$ years, HR 3.2, 95\% CI $2.2,4.5$ ) ( $\geq 40$ years, HR $2.71,95 \%$ CI $1.88,3.91$ ) respectively. A study from Sweden suggested that placental abruption is likely to be common in smokers [25].

\section{Publication bias}

The publication bias was assessed visually using a funnel plot developed by Rev Man 5.3 [22]. The plot was symmetrical indicating a low risk of publication bias (Additional file 2).

\section{Discussion}

This study provides a comprehensive review of the current evidence and estimates of the effect of maternal smoking on the risk of stillbirth. It suggests, women who smoke during pregnancy have a $47 \%$ increased risk of stillbirth and that the risk of stillbirth is more at higher levels of cigarette consumption (Table 2). This effect does not vary according to the gestation at which still birth occurs, however, comparisons between studies would be easier if future work adopted the definition of stillbirth recommended by the WHO [20].

\section{Strengths and limitations}

We implemented a comprehensive search strategy with strict adherence to the protocol, and results were
Table 2 Result summary: maternal smoking in pregnancy and the risk of stillbirth

\begin{tabular}{lll}
\hline Assessment criteria & Odds ratio & $\mathbf{9 5 \% ~ C l}$ \\
\hline $\begin{array}{l}\text { i) Overall pooled Results from main } \\
\text { meta-analysis (25 studies) }\end{array}$ & 1.47 & $1.37-1.57$ \\
$\begin{array}{l}\text { ii) Subgroup analysis by daily amount of } \\
\text { cigarettes consumption }\end{array}$ & & \\
$1-9$ cig/day (6 studies) & 1.09 & $0.97-1.24$ \\
$\geq 10$ cig/day (7 studies) & 1.52 & $1.30-1.78$ \\
iii) Subgroup analysis by gestation period at & & \\
which stillbirth occurred & & \\
Stillbirth $\geq 20$ weeks (11 studies) & 1.43 & $1.32-1.54$ \\
Stillbirth $\geq 24$ week (4 studies) & 1.58 & $1.21-2.06$ \\
Stillbirth $\geq 28$ weeks (7 studies) & 1.33 & $1.18-1.49$ \\
iv) Subgroup analysis by geographic location & & $1.29-1.50$ \\
North America (9 studies) & 1.39 & $1.36-1.78$ \\
Europe (11 studies) & 1.55 & $0.92-1.64$ \\
Asia (1 study) & 1.23 & $1.00-1.96$ \\
South America (1 study) & 1.40 & $1.07-1.55$ \\
Australia (3 studies) & 1.29 & $1.28-1.55$ \\
v) Subgroup analysis by quality & & $1.33-1.67$ \\
High score (14 studies) & 1.41 & \\
Low score (11 studies) & 1.49 & \\
\hline
\end{tabular}

presented in accordance with MOOSE guidelines [19]. Search strategy, data extraction, analysis and quality assessment was performed independently by authors and findings were confirmed within the team. There was no evidence of publication bias. However, the review has some limitations. We could not obtain one study within available timelines [59], and due to limited data presentation, seven studies $[18,42-45,57,58]$ were considered in the narrative synthesis. Secondly, only English language studies were considered mainly for practical purposes and the available timeframe.

Heterogeneity was explored using a variety of subgroup analyses; a high level of heterogeneity was found amongst cohort studies and the pooled estimate derived from these should be treated with some caution. This could be partially attributable to the different factors controlled for in the cohort studies as explained previously. The overall heterogeneity $\left(I^{2}=77 \%\right.$, Figure 2$)$ was higher mainly due to cohort studies, however in case of case control studies $\left(\mathrm{I}^{2}=20 \%\right)$ and cross-sectional studies $\left(\mathrm{I}^{2}=0 \%\right)$, very low levels of heterogeneity was observed. However, this estimate was consistent with synthesised estimates derived from studies with other designs and this consistency suggests it is likely to be valid. It appears that the effect could differ by age $[24,30,38,45,57]$, but data on maternal age was not available in included studies. Study quality was found to be satisfactory. 


\section{Synthesis}

The most recent systematic review by Flenady et al [8] on maternal smoking and the risk of stillbirth, focusing on high-income countries, reported a $36 \%$ increase in the odds of stillbirth (OR 1.36). The review considered cohort and case control studies published between 1998 and 2009 and excluded those which did not control for confounding factors, hospital based studies and took a slightly different approach to dealing with duplicate studies. Acknowledging the difference in the approach, our study is in agreement with the results from Flenady's et al [8] review, and showed a $46 \%$ increased risk of stillbirth (OR 1.46 95\% CI 1.36, 1.55). We included 25 studies in our main meta-analysis, conducted in both developing and developed countries making our findings generalisable. Our review has also analysed the available data according to the definition of stillbirth in gestational weeks, number of cigarettes and study quality, which were not reported in the previous published literature. The review also provides strong evidence that the risk is higher at higher levels of cigarette consumption, indicating a dose related response relationship, outlined in some of the included studies $[15,38,42]$.

The consistency in the size of the effect observed in this study throughout different subgroup analyses and across different study designs, each with their own biases and strengths and limitations, as well as the consistency across studies of differing quality, suggests that this is a true estimate of the size of effect. Further support is obtained from studies where mothers change in smoking behaviour from one pregnancy to another. Hogberg and Cnattingius [14] suggested that mothers who quit smoking from first to second pregnancy reduced their risk of stillbirth to the same level as non-smokers in both pregnancies, while those who smoked in both pregnancies had a 35\% increased risk of stillbirth (OR 1.35) compared to non-smokers.

Our results also provide evidence that the effect of maternal smoking in pregnancy on risk of stillbirth is not dependent on the definition of stillbirth or on the stage of pregnancy at which it occurs. Moreover we did not ascertain, at what point during pregnancy the effect of the exposure is occurring as most studies evaluated the exposure at one point in time only. Previous studies $[26,38]$ suggested that the timing of exposure may influence the risk of stillbirth and in particular [38] suggested that those who quite early in pregnancy may have similar risk to non-smokers. Future studies of this association should measure exposure at different stages of gestation. There are important potential confounders for the effect of maternal smoking on risk of stillbirth, the most important ones are SES, maternal age, maternal weight and medical comorbidities, majority of studies have adjusted for these. Only two studies did not adjust for any confounders $[36,43]$ with no further information.

The exact pathophysiology of fetal exposure to tobacco smoke is not entirely understood, however based on available evidence, possible mechanisms have been conceptualised [30]. Nicotine has been known to cause narrowing of the placental vessels $[60,61]$, which coupled with reduced prostacycline synthesis [62], resulting in increased vascular resistance and consequently impairing blood supply to the fetus [63]. Carbon monoxide in tobacco smoke also reduces fetal oxygenation by forming carboxy-haemoglobin in turn interfering with oxygenation transfer [64]. The correlation between smoking and stillbirth is likely to be explained through increased prevalence of placental complications and fetal growth restriction [14]. The resultant physiological effect of these changes increases the risk of fetal morbidity (small for gestation age and preterm birth) and subsequently may lead to fetal death [30]. Therefore, there is strong biological plausibility for smoking in pregnancy causing stillbirth $[46,65]$.

Recent Cochrane reviews have concluded that behavioural interventions can be effective in helping women to stop smoking in pregnancy [66] even though there remains no evidence for the effectiveness of pharmacotherapy such as NRT in pregnancy [67]. The result of our study suggests that such interventions are important to reduce the risk of stillbirth.

\section{Conclusion}

Our findings support that smoking greatly increases the risk of stillbirth. Smoking in pregnancy is an established cause of a range of pregnancy complications and poor pregnancy outcomes. Every opportunity must be utilised to record smoking status during pregnancy, to give advice and support, including necessary facilities to help women stop smoking. Although we have conducted a comprehensive review, most of the available evidence on the risk from smoking is from developed countries. Smoking is rapidly increasing in the developing world where stillbirth is a major problem; therefore along with focusing on research initiatives, it is important to invest efforts on educating women on the risks of smoking to their unborn child and providing smoking cessation support for pregnant women in these parts of world.

\section{Additional files}

Additional file 1: Supplementary 1. Sample search strategy used for identification of studies.

Additional file 2: Funnel plot.

Abbreviations

NOS: New castle Ottawa scale; SES: Socioeconomic status. 


\section{Competing interests}

The authors declare that they have no competing interests.

\section{Authors' contributions}

The study was conceptualised by SL, TC, TM and AA. All authors participated in electronic search, title screening, study selection, data extraction, analysis and manuscript preparation. AA and TM contributed equally in the manuscript preparation, and thus shared the first authorship equally. All four authors provided intellectual content and approved the manuscript for publication.

\section{Acknowledgments}

We acknowledge the support of the Greenfield Medical Library staff of University of Nottingham, UK.

\section{Author details}

${ }^{1}$ Division of Epidemiology and Public Health, Faculty of Medicine and Health Sciences, University of Nottingham, Clinical Sciences Building 2, Nottingham City Hospital, Hucknall Road, Nottingham NG5 1 PB, UK. ${ }^{2}$ Halo Medical Foundation, Osmanabad, India. ${ }^{3}$ Division of Primary Care, Faculty of Medicine and Health Sciences, University of Nottingham, Nottingham, UK.

Received: 24 October 2014 Accepted: 16 February 2015

Published online: 13 March 2015

\section{References}

1. World Health Organisation $(\mathrm{WHO})$. Recommendations for the prevention and management of tobacco use and second- hand smoke exposure in pregnancy. Geneva: World Health Organization; 2013.

2. Royal College of Physicians. Passive smoking and children: a report of the Tobacco Advisory Group of the Royal College of Physicians. London: Royal College of Physicians; 2013.

3. Royal College of Physicians. Smoking and the young. London: Royal college of Physicians; 1992.

4. Cnattingius S, Bergstrom R, Lipworth L, Kramer MS. Pre-pregnancy weight and the risk of adverse pregnancy outcomes. N Eng J Med. 1998;338:147-52.

5. Fretts RC. Etiology and prevention of stillbirth. Am J Obstet Gynecol. 2005;193:1923-35.

6. Lawn JE, Blencowe H, Pattinson R, Cousens S, Kumar R, Ibiebele I, et al. Stillbirths: Where? When? Why? How to make the data count? Lancet. 2011;377:1448-63.

7. Cnattingius S. The epidemiology of smoking during pregnancy: smoking prevalence, maternal characteristics, and pregnancy outcomes. Nicotine Tob Res. 2004;6:5125-40.

8. Flenady V, Koopmans L, Middleton P, Froen JF, Smith GC, Gibbons K, et al. Major risk factors for stillbirth in high-income countries: a systematic review and meta-analysis. Lancet. 2011;377:1331-40.

9. NHS Information centre. The Infant Feeding Survey 2010. Available at http://www.hscic.gov.uk [Accessed January 2013].

10. Scollo MM, Winstanley MH. Tobacco in Australia: Facts and issues. 4th ed. Melbourne: Cancer Council Victoria; 2012. Available from www.TobaccolnAustralia.org.au.

11. Ebert LM, Fahy K. Why do women continue to smoke in pregnancy? Women Birth. 2007;20:161-8.

12. Wood SL, Chen S, Ross S, Sauve R. The risk of unexplained antepartum stillbirth in second pregnancies following caesarean section in the first pregnancy. BJOG. 2008;115:726-31.

13. Smith GCS, Shah I, White IR, Pell JP, Crossley JA, Dobbie R. Maternal and biochemical predictors of antepartum stillbirth among nulliparous women in relation to gestational age of fetal death. BJOG. 2007;114:705-14.

14. Hogberg L, Cnattingius S. The influence of maternal smoking habits on the risk of subsequent stillbirth: Is there a causal relation? BJOG. 2007;114:699-704.

15. United States Department of Health and Human Services (USDHHS). Health Consequences of Tobacco use among women. In: Women and smoking: a report of the surgeon general. Rockville MD: U.S: Department of Health and Human Services, Centre for Disease Control and Prevention, National Centre for Chronic Disease Prevention and Health Promotion, Office of Smoking and Health; 2001. p. 177-450.
16. Cnattingius S, Haglund B, Meirik O. Cigarette smoking as risk factor for late fetal and early neonatal death. BMJ. 1988;297:258-61.

17. Raymond EG, Cnattingius S, Kiely JL. Effects of maternal age, parity and smoking on the risk of stillbirth. BJOG. 1994;101:301-6.

18. Schramm WF. Smoking during pregnancy: Missouri longitudinal study. Paediatr Perinat Epidemiol. 1997;11:73-83.

19. Stroup DF, Berlin JA, Morton SC, Olkin I, Williamson GD, Rennie D, et al. Meta-analysis of observational studies in epidemiology: a proposal for reporting. JAMA. 2000;283:1-5.

20. Zegers-Hochschild F, Adamson GD, de Mouzon J, Ishihara O, Mansour R Nygren K, et al. The International Committee for Monitoring Assisted Reproductive Technology (ICMART) and the World Health Organization (WHO) Revised Glossary on ART Terminology. Hum Reprod. 2009;24:2683-7.

21. Wells G, Shea B, O'Connell D, Peterson J, Welch V, Losos M, et al. NewcastleOttawa scale (NOS) for assessing the quality of non-randomised studies in meta-analysis. Available from: http://www.ohri.ca/programs/clinical_ epidemiology/oxford.htm (accessed March 10, 2012).

22. Higgins JPT, Green S (editors). Cochrane Handbook for Systematic Reviews of Interventions Version 5.1.0 [updated March 2011]. The Cochrane Collaboration 2011. Available from www.cochrane-handbook.org.

23. Salihu HM, Sharma PP, Getahun D, Hedaytzadeh M, Peters S, Kirby RS, et al. Prenatal tobacco use and risk of stillbirth: a case-control and bidirectional case-crossover study. Nicotine Tob Res. 2008;10:159-66.

24. Ferraz EM, Gray RH. A case-control study of stillbirths in Northeast Brazil. Int J Gynaecol Obstet. 1991;34:3-19.

25. Walles B, Tyden T, Herbst A, Ljungblad U, Rydhstrom H. Maternal health-care program and makers for late fetal death. Acta Obstet Gynecol Scand. 1994;73:773-8

26. Dodds L, King WD, Fell DB, Armson BA, Allen A, Nimrod C. Stillbirth risk factors according to timing of exposure. Ann Epidemiol. 2006;16:607-13.

27. Efkarpidis S, Alexopoulos E, Kean L, Liu D, Fay T. Case-control study of factors associated with intrauterine fetal deaths. MedGenMed. 2004;6:53.

28. Goy J, Dodds L, Rosenberg MW, King W. Health-risk behaviours: examining social disparities in the occurrence of stillbirth. Paediatr Perinat Epidemiol. 2008;22:314-20.

29. Ahlenius I, Thomassen P. The changing panorama of late fetal death in Sweden between 1984 and 1991. Acta Obstet Gynecol Scand. 1999;78:408-14.

30. Aliyu MH, Salihu HM, Wilson RE, Alio AP, Kirby RS. The risk of intrapartum stillbirth among smokers of advanced maternal age. Arch Gynecol Obstet. 2008;278:39-45.

31. Aliyu MH, Salihu HM, Wilson RE, Kirby RS. Prenatal smoking and risk of intrapartum stillbirth. Arch Environ Occup Health. 2007;62:87-92.

32. Gray R, Bonellie SR, Chalmers J, Greer I, Jarvis S, Kurinczuk JJ, et al. Contribution of smoking during pregnancy to inequalities in stillbirth and infant death in Scotland 1994-2003: retrospective population based study using hospital maternity records. BMJ. 2009;339:b3754.

33. Miller EC, Cao HL, Wen SW, Yang Q, Lafleche J, Walker M. The risk of adverse pregnancy outcomes is increased in preeclamptic women who smoke compared with nonpreeclamptic women who do not smoke. Am J Obstet Gynecol. 2010;203:1-8.

34. Mohsin M, Bauman AE, Jalaludin B. The influence of antenatal and maternal factors on stillbirths and neonatal deaths in New South Wales. J Biosoc Sci. 2006;38:643-57.

35. Reddy UM, Laughon SK, Sun L, Troendle J, Willinger M, Zhang J. Prepregnancy risk factors for antepartum stillbirth in the United States. Obstet Gynecol. 2010;116:1119-26.

36. Sutan R, Campbell D, Prescott GJ, Smith WCS. The risk factors for unexplained antepartum stillbirths in Scotland, 1994 to 2003. J Perinatol. 2010;30:311-8.

37. Tuthill DP, Stewart AH, Coles EC, Andrews J, Cartidge PH. Maternal cigarette smoking and pregnancy outcome. Paediatr Perinat Epidemiol. 1999;13:245-53.

38. Wisborg K, Kesmodel U, Henriksen TB, Olsen SF, Secher NJ. Exposure to tobacco smoke in utero and the risk of stillbirth and death in the first year of life. Am J Epidemiol. 2001;154:322-7.

39. Winbo I, Serenius F, Dahlquist G, Kallen B. Maternal risk factors for cause-specific stillbirth and neonatal death. Acta Obstet Gynecol Scand. 2001;80:235-44.

40. Mishra V, Retherford RD, Smith KR. Cooking smoke and tobacco smoke as risk factors for stillbirth. Int J Environ Health Res. 2005;15:397-410. 
41. Robson S, Cameron CA, Roberts CL. Birth outcomes for teenage women in New South Wales, 1998-2003. Aust N Z J Obstet Gynaecol. 2006;46:305-10.

42. Kleinman JC, Pierre MB, Madans Jr JH, Land GH, Schramm WF. The effects of maternal smoking on fetal and infant mortality. Am J Epidemiol. 1988;127:274-82.

43. Raatikainen $\mathrm{K}$, Huurinainen $\mathrm{P}$, Heinonen S. Smoking in early gestation or through pregnancy: a decision crucial to pregnancy outcome. Prev Med. 2007:44:59-63.

44. Huang DY, Usher RH, Kramer MS, Yang H, Morin L, Fretts RC. Determinants of unexplained antepartum fetal deaths. Obstet Gynecol. 2000:95:215-21.

45. Salihu HM, Shumpert MN, Aliyu MH, Alexander MR, Kirby RS, Alexander GR. Stillbirths and infant deaths associated with maternal smoking among mothers aged not greater than or equal to 40 years: a population study. Am J Perinatol. 2004;21:121-9.

46. Moga M, Preda GH. Smoking and pregnancy. J Environ Prot Ecol. 2008:9:566-73.

47. Bukowski R, Carpenter M, Conway D, Coustan D, Dudley DJ, Goldenberg RL, et al. Association between stillbirth and risk factors known at pregnancy confirmation. JAMA. 2011:306:2469-79.

48. Kunzel W, Misselwitz B. Unexpected fetal death during pregnancy - a problem of unrecognised fetal disorders during antenatal care? Eur J Obstet Gynecol Reprod Biol. 2003;110:S86-92.

49. Xu B, Jarvelin MR, Rantakallio P. Maternal smoking in pregnancy and sex differences in perinatal death between boys and girls. Soc Biol. 1998:45:273-7.

50. Kallen $\mathrm{K}$. The impact of maternal smoking during pregnancy on delivery outcome. Eur J Public Health. 2001;11:329-33.

51. Verkerk PH, Buitendijk SE, Verloove-Vanhorick SP. Differential misclassification of alcohol and cigarette consumption by pregnancy outcome. Int J Epidemiol. 1994;23:1218-25.

52. Maleckiene L, Nadisauskiene R, Bergstrom S. Scio-economic demographic and obstetric risk factors for late death of unknown etiology in Luthuania: a case-referent study. Acta Obstet Gynecol Scand. 2001;80:321-5.

53. Beyerleinl A, Von Kriesl R, Hummel M, Lack N, Schiessl B, Giani G. Improvement in pregnancy-related outcomes in the offspring of diabetic mothers in Bavaria, Germany, during 1987-2007. Diabet Med. 2010;27:1379-84.

54. Froen JF, Arnestad M, Vege A, Irgens, Rognum T, Saugstad O. Comparative epidemiology of sudden infant death syndrome and sudden intrauterine unexplained death. Arch Dis Child. 2002;87:118-21.

55. Little RE, Weinberg CR. Risk-factors for antepartum and intrapartum still-birth. Am J Epidemiol. 1993;137:1177-89.

56. McCowan LM, George-Haddad M, Stacey T, Thompson JM. Fetal growth restriction and other risk factors for stillbirth in a New Zealand setting. Aust N Z J Obstet Gynaecol. 2007;47:450-6.

57. Aliyu MH, Salihu HM, Alio AP, Wilson RE, Charkrabarty S, Clayton HB. Prenatal smoking among adolescents and risk of fetal demise before and during labor. J Pediatr Adolesc Gynecol. 2010;23:129-35.

58. Bai J, Wong FW, Gyneshwar R, Stewart HC. Profile of maternal smokers and their pregnancy outcomes in south western Sydney. J Obstet Gynaecol Res. 2000;26:127-32.

59. Flenady V. Causes and risk factors of stillbirth in Australia. Perinatal Society of Austarlia and New Zealand Congress 2008. Gold Coast. Paediatr Child Health. 2008:2-10.

60. Marrow RJ, Ritchie JW, Bull SB. Maternal cigarette smoking: the effects on umbilical and uterine blood flow velocity. Am J Obstet Gynecol. 1988;159:1069-71.

61. Lambers DS, Clark KE. The maternal and fetal physiologic effects of nicotine. Semin Perinatol. 1996;20:115-26.

62. Ylikorkala O, Viinikka L, Lehtovirta P. Effect of nicotine on fetal prostacyclin and thromboxane in humans. Obstet Gynecol. 1985;66:102-5.

63. Bruner JP, Forouzan I. Smoking and Bucally administered nicotine. Acute effect on uterine and umbilical artery Doppler flow velocity wave forms. J Reprod Med. 1991;36:435-40.

64. Salihu HM, Aliyu HM, Kirby RS. In utero nicotine exposure and fetal growth inhibition among twins. Am J Perinatol. 2005;22:421-7.

65. Hammoud AO, Bujold E, Sorokin Y, Schild C, Krapp M, Baumann P. Smoking in pregnancy revisited: findings from a large population-based study. Am J Obstet Gynecol. 2005;192:1856-63.
66. Hajek P, Stead LF, West R, Jarvis M, Hartmann-Boyce J, Lancaster T. Relapse prevention interventions for smoking cessation. Cochrane Database Syst Rev 2013, Issue 8. Art. No.: CD003999. doi:10.1002/14651858.CD003999.pub4.

67. Coleman T, Chamberlain C, Davey MA, Cooper SE, Leonardi-Bee J. Pharmacological interventions for promoting smoking cessation during pregnancy. Cochrane Database Syst Rev 2012, Issue 9. Art. No.: CD010078. doi:10.1002/14651858.CD010078

\section{Submit your next manuscript to BioMed Central and take full advantage of:}

- Convenient online submission

- Thorough peer review

- No space constraints or color figure charges

- Immediate publication on acceptance

- Inclusion in PubMed, CAS, Scopus and Google Scholar

- Research which is freely available for redistribution

Submit your manuscript at www.biomedcentral.com/submit 